\title{
The influence of acute exercise on bone biomarkers: protocol for a systematic review with meta-analysis
}

\author{
E. Dolan ${ }^{1 *}$ D, A. Dumas ${ }^{1}$, K. M. Keane ${ }^{2}$, G. Bestetti ${ }^{1}$, L. H. M. Freitas ${ }^{1}$, B. Gualano ${ }^{1,3}$, W. Kohrt ${ }^{4}$, G. A. Kelley ${ }^{5}$, \\ R. M. R. Pereira ${ }^{6}$, C. Sale ${ }^{7}$ and P. Swinton ${ }^{8}$
}

\begin{abstract}
Background: Bone is a plastic tissue that is responsive to its physical environment. As a result, exercise interventions represent a potential means to influence the bone. However, little is currently known about how various exercise and participant characteristics interact to influence bone metabolism. Acute, controlled, interventions provide an in vivo model through which the acute bone response to exercise can be investigated, typically by monitoring circulating bone biomarkers. Currently, substantial heterogeneity in factors such as study design, quality, exercise, and participant characteristics render it difficult to synthesize and evaluate the available evidence. Using a systematic review and meta-analytic approach, the aim of this investigation is to quantify the effect of an acute exercise bout on circulating bone biomarkers as well as examine the potential factors that may moderate this response, e.g., variation in participant, exercise, and sampling characteristics.
\end{abstract}

Methods: This protocol was designed in accordance with the PRISMA-P guidelines. Seven databases (MEDLINE, Embase, Sport Discus, Cochrane CENTRAL, PEDro, LILACS, and Ibec) will be systematically searched and supplemented by a secondary screening of the reference lists of all included articles. The PICOS (Population, Intervention, Comparator, Outcomes and Study Design) approach was used to guide the determination of the eligibility criteria. Participants of any age, sex, training, or health status will be considered for inclusion. We will select studies that have measured the bone biomarker response before and after an acute exercise session. All biomarkers considered to represent the bone metabolism will be considered for inclusion, and sensitivity analyses will be conducted using reference biomarkers for the measurement of bone resorption and formation (namely $\beta$ CTX-1 and P1NP). Multi-level, meta-regression models within a Bayesian framework will be used to explore the main effect of acute exercise on bone biomarkers as well as potential moderating factors. The risk of bias for each individual study will be evaluated using a modified version of the Downs and Black checklist while certainty in resultant outcomes will be assessed using the Grading of Recommendations Assessment, Development and Evaluation (GRADE) approach.

\footnotetext{
* Correspondence: eimeardolan@usp.br

${ }^{1}$ Applied Physiology and Nutrition Research Group, School of Physical

Education and Sport; Faculdade de Medicina FMUSP, University of Sao Paulo,

Sao Paulo, Brazil

Full list of author information is available at the end of the article
}

\section{$\triangle B M C$}

(c) The Author(s). 2020 Open Access This article is licensed under a Creative Commons Attribution 4.0 International License, which permits use, sharing, adaptation, distribution and reproduction in any medium or format, as long as you give appropriate credit to the original author(s) and the source, provide a link to the Creative Commons licence, and indicate if changes were made. The images or other third party material in this article are included in the article's Creative Commons licence, unless indicated otherwise in a credit line to the material. If material is not included in the article's Creative Commons licence and your intended use is not permitted by statutory regulation or exceeds the permitted use, you will need to obtain permission directly from the copyright holder. To view a copy of this licence, visit http://creativecommons.org/licenses/by/4.0/ The Creative Commons Public Domain Dedication waiver (http://creativecommons.org/publicdomain/zero/1.0/) applies to the data made available in this article, unless otherwise stated in a credit line to the data. 
(Continued from previous page)

Discussion: A better understanding of the bone metabolic response to an acute bout of exercise has the potential to advance our understanding of the mechanisms through which this stimulus impacts bone metabolism, including factors that may moderate this response. Additionally, we will identify current gaps in the evidence base and provide recommendations to inform future research.

Systematic review registration: This protocol was prospectively registered in the Open Science Framework Registry (https://osf.io/6f8dz)

Keywords: Exercise, Bone, Meta-analysis, Systematic review

\section{Background}

Bone is a plastic tissue that is responsive to its physical environment. As a result, exercise interventions have the potential to impact bone via a range of direct and indirect mechanisms [1-3]. These include the direct impact of physical activity-induced loading cycles [4], activityspecific metabolic signals such as alterations to calcium kinetics [5], redox balance [6], $\mathrm{pH}$ perturbations [7], and indirect signals mediated via other tissues, primarily skeletal muscle [8]. Substantial research and public resources are expended on investigating and implementing exercise-based interventions for populations who are susceptible to bone-related disorders. In general, activities that convey higher-impact, multi-directional, and unaccustomed loading patterns are considered to convey the greatest osteogenic stimulus, and for this reason, guidelines for the use of exercise to improve bone generally recommend that both resistance and impact-based modalities are employed [9-11]. This approach has been reported to be effective in many populations, with metaanalytic data reporting a positive effect of controlled exercise interventions on bone density in a range of populations that include pre- [12] and postmenopausal [13] women, older adults [14], individuals with osteoporosis [15], and children [16]. However, it is important to understand that several of the aforementioned metaanalyses included a number of studies that reported no effect of exercise on the bone, while some studies even suggest that the bone may be negatively impacted by very high volumes or intensities of exercise, e.g., in athletes competing in sports that emphasize leanness or those that rely upon repetitive loading cycles [17-20].

Currently, there is a lack of understanding regarding the mechanistic pathways through which the bone responds to exercise. As a result, the identification of what combination of participant and exercise characteristics determine whether an osteogenic, osteoneutral, or even an osteolytic effect will be induced remains elusive. Acute exercise interventions are commonly used as an in vivo model to investigate the bone response to exercise, and these types of interventions have much to contribute to advancing the understanding of the processes through which the bone responds to exercise (and other acute stimuli). Bone remodeling is the dominant process through which mature bone responds to exercise [21], and it comprises a sequential and synchronized process of bone activation, resorption, reversal, and formation $[3,22]$. Circulating bone biomarkers, which are widely used in the clinical setting [23-25], are used to provide information on the dynamic state of bone remodeling. This is important because static indicators of bone health and function, such as bone mass measured by DXA, or microarchitecture as indicated by computed tomography or magnetic resonance imaging, are slow to respond to stimuli, with measurable changes taking months or even years to occur [26]. As a result, bone biomarkers which are capable of providing more immediate information on the current bone state are the only viable option available to evaluate the impact of controlled, acute, exercise interventions on bone metabolism and, thus, to help identify the pathways that influence this response.

Recently, our research group published a comprehensive narrative review; the intention of which was to synthesize and evaluate our current understanding of the bone metabolic response to exercise [21]. In that review, we observed that a single exercise session often elicits an increase in biomarkers that are indicative of bone resorption [27-30], indicating an initial catabolic response of bone to exercise. In contrast, longer-term adaptations to exercise training are often characterized by an increase in bone formation markers [31-39] that occur concurrently with positive changes in the bone mass or microarchitecture [36, 40-42]. These observations appear plausible when considered in the context of what is suggested about the bone remodeling cycle, whereby the initial osteoclastic activation may be required to trigger a subsequent increase in osteoblastic activity [22]. In order to further advance our understanding beyond these general observations, it is necessary to progress beyond dichotomous interpretations of increases/decreases/no changes in isolated bone biomarkers and instead consider the magnitude and context of the reported changes. However, this is challenging as considerable heterogeneity in research findings exists, most likely due to the large variation in the design, 
characteristics, and quality of available studies. Appropriate systematic review protocols and meta-analytic models are essential to overcome these challenges. However, to the best of our knowledge, no systematic review, with or without meta-analysis, has been conducted on this topic. Thus, the aim of this investigation is to use the systematic review and meta-analytic approach to quantify the effect of an acute bout of exercise on circulating biomarkers indicative of the bone metabolism, as well as investigate potential the factors that may moderate this response, e.g., variation in participant, exercise, and sampling characteristics.

\section{Methods}

\section{Overview}

The protocol for this review adheres to previously published guidelines [43] and includes all items described in the checklist of Preferred Reporting Items for Systematic Review and Meta-Analysis Protocols (PRISMA-P) [44]. The completed checklist is available in Supplementary File 1, and the protocol for this review was preregistered in the Open Science Framework Registry (https://osf.io/6f8dz).

\section{Eligibility criteria}

The PICOS (Population, Intervention, Comparator, Outcomes and Study Design) approach was used to guide the determination of the eligibility criteria for this review.

\section{Population}

In order to allow for the analysis of whether participant characteristics (sex, age, health or training status) will impact the bone biomarker response to a single bout of exercise, men and women of any age and health or training status will be considered for inclusion in this review.

\section{Intervention}

Studies that investigate a single exercise bout of any duration, intensity, or type will be considered for inclusion. This includes all interventions that require an active effort by the individual (including movement therapies such as yoga and tai chi), whereby passive interventions (e.g., vibration therapy) will be excluded. Exercise interventions will be categorized according to their type (e.g., resistance, aerobic, multi-modal, plyometric, calisthenics), duration (min), intensity (e.g., percentage of maximum capacity), total work (defined as duration $\times$ intensity), and impact level (i.e., high-impact/multi-directional; low-impact/repetitive; moderate-impact/repetitive; or low-impact with high muscular load). A more detailed explanation of these categories along with examples is provided in the codebook described in Supplementary File 2.
Given that bone biomarkers are acutely responsive to nutrient intake and status [45-47], a secondary analysis of studies that investigate how nutritional strategies influence the bone biomarker response to exercise will be conducted. For studies that investigate exercise interventions with and without a specific nutritional intervention (e.g., calcium [27] or carbohydrate [48] supplementation), data from the non-nutritional condition will be used in the main meta-analysis while the nutritionally manipulated condition will be used to inform this secondary analysis. In addition, whether the exercise bout is conducted in a fed or fasted state will be considered.

\section{Comparator}

The primary comparison of interest is the pre-post difference in the bone biomarkers as a result of an acute exercise bout. Estimation of typical error in the bone biomarker assessments across the different time periods will be assessed using data from any of the eligible studies that include a non-exercise control condition as well as available data on circadian variation in bone biomarkers $[47,49]$. This data will be accounted for within the meta-analytic model for the purpose of providing a more precise estimate of the true effect of exercise on the bone. In addition, sensitivity analysis limited to those studies that report data for a non-exercise control group will be conducted and compared to the results of the main model to identify whether the exclusion of a nonexercise control group, as is common in these types of studies, meaningfully influences effect size estimation.

\section{Outcomes}

The primary outcome of interest is the bone biomarker response to an acute exercise bout. Bone biomarkers will be considered in relation to the processes of (1) bone formation (bone-specific alkaline phosphatase (B-ALP), dickkopf-1 (DKK-1), carboxyterminal propeptide of type 1 procollagen ( $\mathrm{P} 1 \mathrm{CP}), \mathrm{N}$-terminal propeptide of type 1 procollagen (P1NP), and sclerostin), (2) bone resorption (pyridinoline (Pyr), deoxypyridinoline (Dpd), carboxyterminal telopeptide of type 1 procollagen (ICTP), aminoterminal telopeptide of type 1 collagen (NTx), cathepsin $\mathrm{K}, \mathrm{C}$-terminal telopeptide of type 1 collagen $(\beta-C T X-1)$, tartrate resistance acid phosphatase isoenzyme 5b (TRAP5b), ratio of osteoprotegerin to receptor activator NF kappaB ligand (OPG/RANKL), hydroxylysine, and hydroxyproline), and (3) general bone remodeling (osteopontin and total and undercarboxylated osteocalcin (T/U-OC)). These are all commonly reported biomarkers that have been suggested to measure the processes of bone metabolism, although some are nonspecific to the bone and/or are difficult to accurately measure [21]. For this reason, we will conduct sensitivity analyses using only those markers that have been 
designated as reference markers for the assessment of bone formation (P1NP) and resorption ( $\beta$-CTX-1). These particular biomarkers were chosen based upon their relatively high specificity to the bone metabolism, their relatively small biological variability, and their responsiveness to osteogenic intervention [23-25, 50]. An additional sensitivity analysis will also be undertaken using all bone biomarkers apart from B-ALP, as it is suggested to represent late-stage bone mineralization and, as such, is unlikely to respond to a single exercise bout. Biomarkers indicative of calcium metabolism (circulating ionized or albumin-adjusted calcium, phosphorus, and parathyroid hormone) will be considered a secondary outcome. In the case of multiple available outcomes due to repeated sampling, the pre-exercise sample that is taken closest to the start of the exercise bout will be selected as the baseline value, while all sampling points during and post-exercise will be extracted and used to model the time course of the observed bone biomarker changes.

Fixed effects related to the study, participants, sampling, and exercise characteristics will be incorporated into the model to identify potential factors that may influence the main effect of acute exercise on bone biomarkers. These include (1) participant characteristics (age, sex, training status, health status), (2) exercise characteristics (type, duration, intensity, total work done, impact level), and (3) blood sampling characteristics (nutritional status, assay type, sample timing). A more detailed description of all data that will be extracted is described in the accompanying codebook (Supplementary File 2).

\section{Outcome prioritization}

As described in our recent narrative review, it appears that total work performed during the exercise bout, i.e., higher intensity efforts conducted for longer periods of time, may have a greater influence on the bone biomarker response than either intensity or duration alone [21]. Therefore, we hypothesize that this factor will be the most influential moderator of the main effect. In contrast to the commonly held view that impact is required to elicit an osteogenic response [2], it appears that low-impact, repetitive loading exercises such as cycling regularly elicit a bone biomarker response $[5,27$, 51], indicating that exercise type and impact level may not exert a strong influence on the main effect. In relation to participant characteristics, age is likely to be an important factor determining the bone biomarker response to exercise given that children and young adults are thought to have greater bone plasticity, while older adults may experience age-related osteogenic resistance [52]. It is unlikely that sufficient data will be available to investigate the independent influence of each of these stated factors, e.g., most of the studies conducted to date have used young, healthy, men. As a result, there may be insufficient data on older adults, or women, to allow for the analysis of the moderating influence of these factors. When insufficient data is available for the analysis (defined as a minimum of four data points per group for categorical variables or 10 data points for continuous variables [53]), these will be suggested as potential directions for future research.

\section{Study design}

Any study design that includes measurement of bone biomarkers before and after an acute bout of exercise will be considered for inclusion. These include randomized and non-randomized controlled trials, including cross-over trials, as well as single group pre-post studies. Our rationale for including these different designs is based on (1) our desire to be as inclusive as possible, (2) to identify if the results are associated with these different designs, and (3) provide direction for future research with respect to designing studies aimed at examining the effects of an acute bout of exercise on bone biomarkers. Natural experiments, i.e., studies that measure bone biomarkers before and after real-life athletic events (e.g., endurance events) will also be considered for inclusion; however, because these investigations are prone to substantially more variation than experimental laboratory studies, they will not be included in the main analysis, and instead, a secondary analysis will be undertaken using these data.

\section{Information sources}

Seven electronic databases, namely MEDLINE, Embase, Cochrante CENTRAL, Sport Discus, PEDro, LILACS, and IBEC will be used to source material for this review. MEDLINE, Embase, and Cochrane CENTRAL were selected based on the recommendations of the Cochrane Collaboration Handbook for Systematic Reviews of Interventions [54]. MEDLINE and Embase will be accessed using the OVID platform. Sport Discus and PEDro will be searched based on their specific relevance for this topic area, while the LILACS and IBECS databases will be accessed via the Virtual Health Library portal in order to identify Latin American- and Caribbean-based literature as well as health science journals published in Spanish. The primary database search strategy will be supplemented by citation screening of all studies included in the review along with relevant reviews and book chapters (e.g., Banfi et al. [55], Dolan et al. [21], and Alp [56]).

\section{Search strategy}

All searches will be conducted by ED. Free-text terms related to each of the core concepts to be explored in 
this review will be used in each of the aforementioned databases. These include bone AND (exercise OR physical activity) AND (biomarkers OR turnover OR remodeling OR formation OR resorption). A combination of free-text and database-specific subject headings will be used to more comprehensively assess all available studies. The MEDLINE and Embase searches will be conducted using the OVID platform, and the advanced search multi-purpose $(\mathrm{mp})$ function will be used. This function simultaneously searches several fields, including mapping free-text terms to the relevant database-specific subject headings, namely MeSH headings for MEDLINE and Emtree for Embase. Searches will be limited to human studies, and no restrictions will be placed on either date or language. Our research team comprises native English, Portuguese, and Spanish speakers. Eligible studies in any other language will be translated into English using feely available online translators (Google Translate or Babelfish). If a potentially eligible article cannot be adequately translated, we will contact the study authors for clarification, and if no response is obtained the article will be excluded from the review. Only peer-review studies published in scientific journals will be considered for inclusion in this review. Unpublished work, defined as master's theses, dissertations, abstracts from conference proceedings, and technical reports will not be included. Our rationale is based on the work of van Driel et al. [57] who concluded that (1) the difficulty in retrieving unpublished work could lead to selection bias, (2) many unpublished trials are eventually published, (3) the methodological quality of such studies is poorer than those that are published, and (4) the effort and resources required to obtain unpublished work may not be warranted. In line with Cochrane Collaboration recommendations [54], the full search strategy for the MEDLINE search was submitted for peer review to an information scientist using the Peer Review for Electronic Search Strategy (PRESS) Guideline Assessment form [58] and is available in Supplementary File 3. This search strategy will be replicated for each of the other databases, and the individual search strategies will be reported as a supplementary file to the final manuscript. Search results from each database will be downloaded as a ris file then uploaded to a systematic review management software (covidence.org) and deduplicated using the automatic option provided therein. In the case that any duplicate records are not detected using this automatic option, they will be manually removed during the screening process.

\section{Study records}

\section{Study selection}

A three-stage selection strategy will be independently undertaken by two members of the review team (KK and
ED; title/abstract screen; full-text screen/full-text appraisal), and the results will be filtered using the eligibility criteria described above. The independent screeners will not be blinded to any study information and will convene at the end of each screening stage to resolve any discrepancies. These discrepancies will be resolved by a discussion, with a third party invited to mediate if required. During the full-text screen and review stages, reasons for exclusion will be categorized as one or more of the following: (1) inappropriate population, (2) inappropriate intervention, (3) inappropriate comparator, (4) inappropriate outcome, (5) inappropriate study design, and (6) others. The search strategy will be schematically illustrated using the PRISMA search flow diagram (see Fig. 1).

\section{Data extraction}

Data will be independently extracted by 2 members of the review team (AD/LHMF and $\mathrm{ED} / \mathrm{GB})$ into 2 independent pre-piloted excel sheets. All data will be independently coded by $\mathrm{AD}$ and $\mathrm{ED}$ as described in the accompanying codebook (Supplementary File 3). Prior to compiling all data into a single master sheet, $\mathrm{AD}$ and $\mathrm{ED}$ will meet to review all selections for agreement. Discrepancies will be resolved by a consensus, and if consensus cannot be reached, a third member of the review team will be invited to mediate. Prior to correcting disagreements, the overall agreement rate will be calculated using the Cohens $K$ statistic [59]. If all required data is not available in the published article, study authors will be contacted by ED to request additional information (maximum of two e-mail attempts). If the primary outcome data is not available (either from the original paper or on request from the authors), then the paper will be excluded from the study. Our hierarchical random effects model means that results from studies can be included even if some secondary data is missing, and so studies will only be excluded if the primary data is not available, i.e., data on bone biomarkers recorded before and after an acute exercise session.

\section{Risk of bias assessment in individual studies}

The risk of bias for each individual study will be independently assessed in duplicate by KK and AD/ED using a modified version of the Downs \& Black checklist [50]. This tool was chosen over others as it provides a comprehensive assessment of the methodological quality of both randomized and non-randomized trials in healthcare research and has been validated as a tool to ascertain quality of reporting as well as internal and external validity [60]. Some items in the original tool were deemed unnecessary for this review, either because they were specifically relevant to longitudinal interventions and therefore not required in an investigation on the 


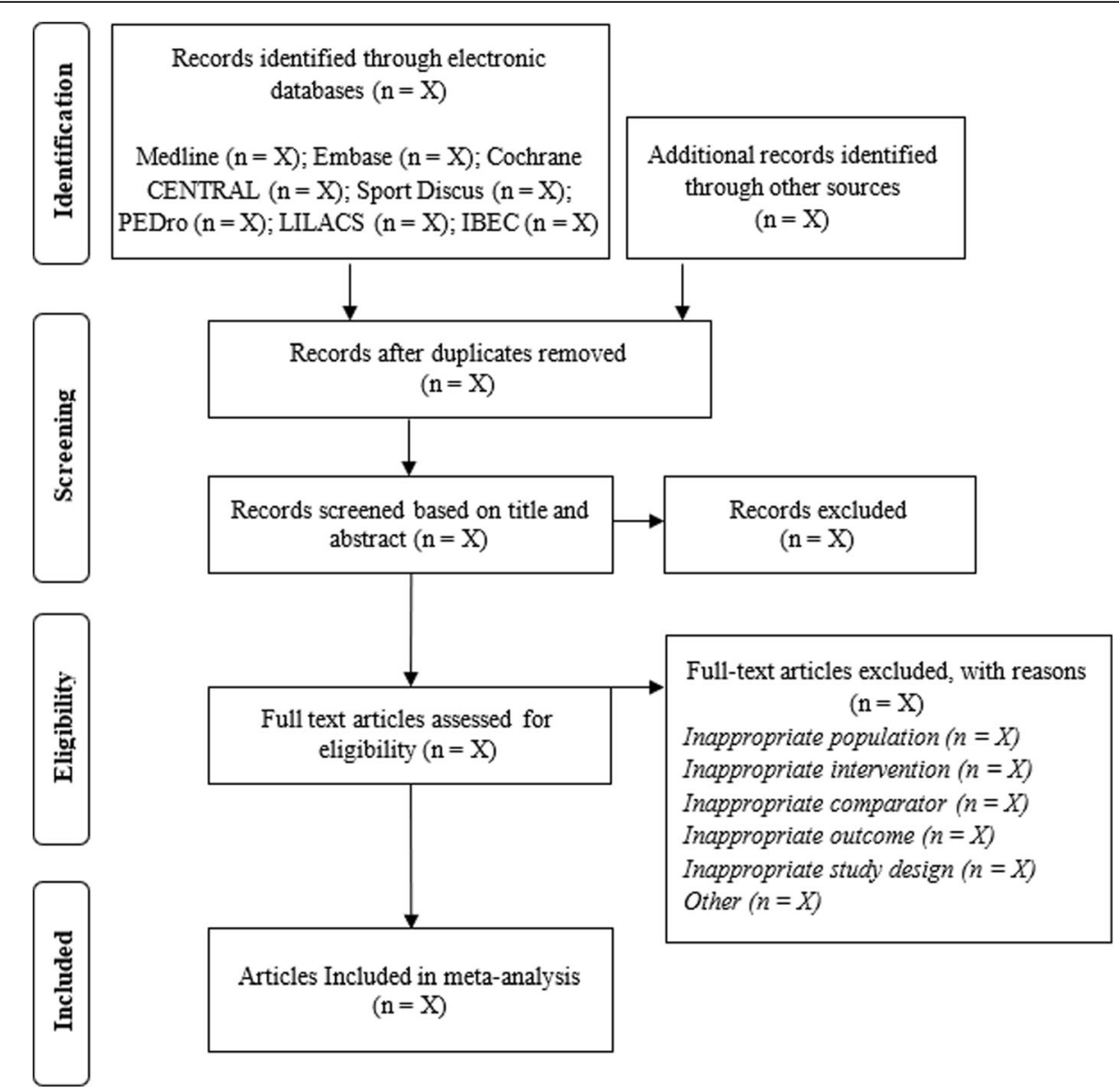

Fig. 1 PRISMA flow diagram depicting the search process

biomarker response to an acute exercise bout or because they related to the quality of reporting on factors that were not deemed to potentially bias the specific outcomes of interest in this review. The modified tool is available in Supplementary File 4. The results of this assessment will not be used to exclude any eligible studies. Rather, it will contribute to ascertaining confidence in the cumulative evidence of obtained results as well as highlight specific areas that future investigations on this topic should address to improve the quality of future research.

\section{Data synthesis}

A Bayesian framework was chosen over a frequentist approach as it provides a more flexible modeling approach that will enable results to be interpreted intuitively through reporting of subjective probabilities [61]. The effect of exercise on bone biomarkers will be quantified by the effect sizes calculated with standardized mean differences pre- and post-exercise. Three-level randomeffects Bayesian hierarchical models will be used to pool effect sizes and model average effects, variance within studies, variance between studies, and covariance of multiple outcomes reported in the same study (e.g., multiple bone biomarkers and/or single bone biomarker reported at multiple post-exercise time points). Withinstudy variance is influenced by pre-post correlations [62] that are generally not reported. Primary data obtained from relevant studies (including that produced in the laboratories of the study team) will be used to develop informative priors to model these within-study variances. Non-informative priors will be used for all other model parameters. Inconsistency in models will be described by comparing variances across the three levels. Inferences from all analyses will be performed on posterior samples generated using the Hamiltonian Markov Chain Monte Carlo method and through the use of credible intervals and calculated probabilities. Interpretations will be based on the range of values within the credible intervals and calculation of probabilities that the magnitude of the average effect size exceeds commonly used qualitative thresholds (e.g., small (0.2), medium (0.5), large (0.8) [63]). Analyses will be performed using the $\mathrm{R}$ wrapper package brms interfaced with Stan to perform the 
sampling [64]. The primary meta-analyses will comprise three univariate models with outcomes categorized as (1) bone formation, (2) bone resorption, and (3) bone remodeling. Sensitivity analyses will be conducted with restriction to reference markers for bone formation and resorption (P1NP and $\beta$-CTX-1). When possible, metaregression will be used to explore the effect of various potential moderators as described above in the "Outcomes" section. Meta-regression will be performed when there is sufficient data including a minimum of four data points per category level or 10 data points for continuous variables [53]. Small-study effects (publication bias, etc.) will be visually inspected with funnel plots and quantified with a multi-level extension of Egger's regression-intercept test [65].

\section{Confidence in cumulative evidence}

The strength of evidence will be independently assessed in duplicate by ED and AD using the Grading of Recommendations Assessment Development and Evaluation (GRADE) instrument [66]. Potential downgrading factors include risk of bias, inconsistency, indirectness, imprecision, or the presence of publication bias. The risk of bias will be assessed using a modified Downs and Black checklist as described above, with the median assessment used to describe the resultant outcomes. In relation to the directness of outcomes, any data point that does not include a non-exercise control group will be downgraded a level. Median ratings from the risk of bias and indirectness assessments will be used to cumulatively describe these assessments for each outcome described in the review. Consistency will be ascertained using the meta-analysis results, and based on visual inspection of the effect size estimates, whether or not confidence intervals overlap, and on statistical tests for heterogeneity. Precision will be judged based on the number of outcomes available and on visual analysis of the width of the confidence intervals. Small-study effects (publication bias, etc.), will be assessed using Egger's regression-intercept test along with visual inspection of funnel plots. Potential upgrading factors included the presence of large effects, evidence of dose-response, and the presence of plausible residual confounding factors.

\section{Conclusions}

A better understanding of the bone metabolic response to acute exercise has the potential to advance our understanding of the mechanisms through which this stimulus impacts bone metabolism. The comprehensiveness of the review will also allow identification of current gaps in the evidence base and subsequent recommendations to inform future research. Broadly, these recommendations will include the identification of pertinent research questions that are currently under-studied as well as the highlighting of methodological issues that were apparent across the evidence base as a whole, and which should be corrected to improve future research efforts. For example, although this analysis will consider all biomarkers that are suggested to effect bone metabolism, many of these are non-specific to the bone and/or are difficult to measure. As a result, we expect that the current analysis will provide insight as to which of these markers are most likely to effect exercise-induced changes in bone metabolism and, thus, will help to guide future research in this area. Finally, the results of this investigation will be disseminated via a presentation at relevant conferences and publications in peer-reviewed journals, serving as a contemporary foundation on which on-going research efforts in this topic can be based.

\section{Supplementary Information}

The online version contains supplementary material available at https://doi. org/10.1186/s13643-020-01551-y.

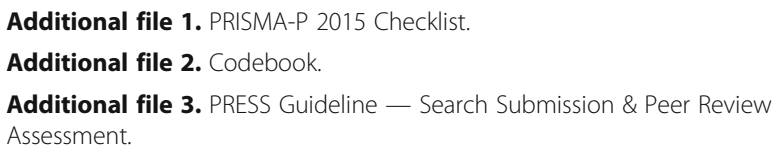

Additional file 4. Modified Downs \& Black checklist.

\section{Abbreviations}

BMU: Basic multicellular unit; DXA: Dual-energy X-ray absorptiometry; PRIS MA-P: Preferred Reporting Items for Systematic Review and Meta-Analysis Protocols; OSF: Open Science Framework; PICOS: Population, Intervention, Comparator, Outcomes and Study Design; B-ALP: Bone-specific alkaline phosphatase; DKK-1: Dickkopf-1; P1CP: Carboxyterminal propeptide of type 1 procollagen; P1NP: N-terminal propeptide of type 1 procollagen;

Pyr: Pyridinoline; Dpd: Deoxypyridinoline; ICTP: Carboxyterminal telopeptide of type 1 procollagen; NTx: Aminoterminal telopeptide of type 1 collagen; $\beta$ CTX-1: C-terminal telopeptide of type 1 collagen; TRAP5b: Tartrate resistance acid phosphatase isoenzyme 5b; OPG: Osteoprotegerin; RANKL: Receptor activator NF kappaB ligand; T/U-OC: Total and undercarboxylated osteocalcin; RIS: Research information systems; BMI: Body mass index; GRADE: Grading of Recommendations Assessment Development and Evaluation

\section{Acknowledgements}

Not applicable

\section{Authors' contributions}

ED, PAS, CS, and GK designed the protocol, with content specific critical input from WK, BG, and RMRP. ED will conduct all database searches. Study screening and selection will be done by ED and KK. Data extraction will be done by ED/GB and AD/LMHF, and the extracted data will be coded by ED and $A D . K K$ and ED/AD will evaluate all studies for risk of bias, while certainty in resultant outcomes will be assessed by ED and AD. Any discrepancies between the independent assessments will be resolved by a discussion, and if consensus cannot be reached, a third member of the research team (CS or PAS) will be invited to mediate. PAS will undertake the statistical analysis, with critical input from GK. ED will write the first draft of the resultant manuscript, with ongoing critical input from other authors. All authors have read and agreed with this protocol. ED is the guarantor.

Authors' information

Not applicable. 


\section{Funding}

Eimear Dolan is supported by a research grant from the Fundação de Amparo à Pesquisa do Estado de São Paulo (FAPESP)/UK Academies Young Investigator Program (2019/05616-6 and 2019/26899-6).

\section{Availability of data and materials}

All data generated or analyzed during this study will be included in the published article and its supplementary files. Should any additional information be required, it will be made available from the corresponding author on reasonable request.

\section{Ethics approval and consent to participate} Not applicable.

\section{Consent for publication}

Not applicable

\section{Competing interests}

The authors declare that they have no competing interests.

\section{Author details}

${ }^{1}$ Applied Physiology and Nutrition Research Group, School of Physical Education and Sport; Faculdade de Medicina FMUSP, University of Sao Paulo, Sao Paulo, Brazil. ${ }^{2}$ Department of Sport, Exercise and Rehabilitation, Northumbria University, Newcastle upon Tyne, UK. ${ }^{3}$ Food Research Centre, University of São Paulo, Sao Paulo, SP, Brazil. ${ }^{4}$ Centre for Women's Health Research, School of Medicine, University of Colorado, Aurora, USA. ${ }^{5}$ Department of Biostatistics, West Virginia University, Morgantown, USA. ${ }^{6}$ Bone Metabolism Laboratory, Rheumatology Division, Faculdade de Medicina FMUSP, Universidade de Sao Paulo, Sao Paulo, SP, Brazil.

${ }^{7}$ Musculoskeletal Physiology Research Group, Sport, Health and Performance Enhancement (SHAPE) Research Centre, School of Science and Technology, Nottingham Trent University, Nottingham, UK. ${ }^{8}$ School of Health Sciences, Robert Gordon University, Aberdeen, UK

\section{Received: 21 July 2020 Accepted: 30 November 2020}

\section{Published online: 12 December 2020}

\section{References}

1. Bass $S$, Eser P, Daly R. The effect of exercise and nutrition on the mechanostat. J Musculoskelet Neuronal Interact. 2005;5(3):239-54.

2. Frost H. A 2003 update of bone physiology and Wolff's law for clinicians. Angle Orthod. 2004;74(1):3-15.

3. Robling $\mathrm{AG}$, Castillo $\mathrm{AB}$, Turner $\mathrm{CH}$. Biomechanical and molecular regulation of bone remodeling. Annu Rev Biomed Eng. 2006;8(1):455-98.

4. Orr A, Helmke B, Blackman B, Schwartz M. Mechanisms of mechanostransduction. Dev Cell. 2006;10(1):11-20.

5. Kohrt W, Wherry S, Wolfe P, Sherk D, Wellington T, Swanson C, et al. Maintenance of serum ionized calcium during exercise attenuates parathyroid hormone and bone resorption responses. J Bone Miner Res. 2018:33(7):1326-34.

6. Ha H, Bok Kwak H, Woong Lee S, Mi Jin H, Kim HM, Kim HH, et al. Reactive oxygen species mediate RANK signaling in osteoclasts. Exp Cell Res. 2004; 301(2):119-27

7. Krieger NS, Frick KK, Bushinsky DA. Mechanism of acid-induced bone resorption. Curr Opin Nephrol Hypertens. 2004;13(4):423-36.

8. Tagliaferri C, Wittrand Y, Davicco M, Walrand S, Coxam V. Muscle and bone, two interconnected tissues. Ageing Res Rev. 2015;21:55-70.

9. Turner $\mathrm{CH}$, Robling $\mathrm{AG}$. Designing exercise regimens to increase bone strength. Exerc Sport Sci Rev. 2003;31(1):45-50.

10. Beck B, Daly R, Fiatarone Singh M, Taaffe DR. Exercise and Sports Science Australia (ESSA) position statement on exercise prescription for the prevention and management of osteoporosis. J Sci Med Sport. 2017;20(5): 438-45.

11. National Osteoporosis Society. Strong Steady Straight. An Expert Consensus Statement on physical activity and exercise for osteoporosis. 2019. https:// www.bgs.org.uk/sites/default/files/content/attachment/2019-02-20/FINAL\%2 0Consensus\%20Statement_Strong\%20Steady\%20and\%20Straight_DEC18. pdf.
12. Kelley G, Kelley K, Kohrt W. Exercise and bone mineral density in premenopausal women: a meta-analysis of randomized controlled trials. Int J Endocrinol. 2013;741639.

13. Howe T, Shea B, Dawson L, Downie F, Murry A, Ross C, et al. Exercise for preventing and treating osteoporosis in postmenopausal women. Cochrane Database Syst Rev. 2011:6(7):CD000333.

14. Marques E, Mota J, Carvalho J. Exercise effects on bone mineral density in older adults: a meta-analysis of randomized controlled trials. Age (Omaha). 2012;34(6):1493-515.

15. Hagen K, Dagfinrud H, Moe R, Osteras N, Kjeken I, Grotle M, et al. Exercise therapy for bone and muscle health: an overview of systematic reviews. BMC Med. 2012;10(1):167.

16. Hind K, Burrows M. Weight-bearing exercise and bone mineral accrual in children and adolescents: a review of controlled trials. Bone. 2007;40(1):14-

17. Olmedillas H, Gonzalez-Aquero A, Moreno L, Casajus J, Vicente-Rodríguez G. Cycling and bone health: a systematic review. BMC Med. 2012;10:168.

18. Scofield K, Hecht S. Bone health in endurance athletes: runners, cyclists, and swimmers. Curr Sports Med Rep. 2012;11(6):328-34.

19. Dolan E, McGoldrick A, Davenport C, Kelleher G, Byrne B, Tormey W, et al. An altered hormonal profile and elevated rate of bone loss are associated with low bone mass in professional horse-racing jockeys. J Bone Miner Metab. 2012:30(5):534-42.

20. Ackerman K, Nazem T, Chapko D, Russell M, Mendes N, Taylor A, et al. Bone microarchitecture is impaired in adolescent amenorrheic athletes compared with eumenorrheic athletes and nonathletic controls. J Clin Endocrinol Metab. 2011;96(10):3123-33.

21. Dolan E, Varley I, Ackerman K, Pereira R, Elliott-Sale K, Sale C. The bone metabolic response to exercise and nutrition. Exerc Sport Sci Rev. 2020; 48(2):49-58.

22. Hadjidakis D, Androulakis I. Bone remodeling. Ann N Y Acad Sci. 2006;1092: 385-96.

23. Vasikaran SD, Paul Chubb SA. The use of biochemical markers of bone turnover in the clinical management of primary and secondary osteoporosis. Endocrine. 2016;52(2):222-5.

24. Chubb SAP, Byrnes E, Manning L, Golledge J, Ebeling PR, Flicker L, et al. Bone turnover markers: defining a therapeutic target. Clin Biochem. 2017; 50(3):162-3.

25. Bauer D, Krege J, Lane N, Leary E, Libanati C, Miller P, et al. National Bone Health Alliance Bone Turnover Marker Project: current practices and the need for US harmonization, standardization, and common reference ranges. Osteoporos Int. 2012;23(10):2425-33.

26. Eriksen E. Cellular mechanisms of bone remodeling. Rev Endocr Metab Disord. 2010;11(4):219-27.

27. Barry DW, Hansen KC, Van Pelt RE, Witten M, Wolfe P, Kohrt WM. Acute calcium ingestion attenuates exercise-induced disruption of calcium homeostasis. Med Sci Sports Exerc. 2011:43(4):617-23.

28. Guillemant J, Accarie C, Peres G, Guillemant S. Acute effects of an oral calcium load on markers of bone metabolism during endurance cycling exercise in male athletes. Calcif Tissue Int. 2004;74(5):407-14.

29. Scott JPR, Sale C, Greeves JP, Casey A, Dutton J, Fraser WD. Effect of fasting versus feeding on the bone metabolic response to running. Bone. 2012 51(6):990-9.

30. Rantalainen $T$, Heinonen A, Linnamo V, Komi P, Takala T, Kainulainen H. Short-term bone biochemical response to a single bout of high-impact exercise. J Sport Sci Med. 2009;8(4):553-9.

31. Adami S, Gatti D, Viapiana O, Fiore CE, Nuti R, Luisetto G, et al. Physical activity and bone turnover markers: a cross-sectional and a longitudinal study. Calcif Tissue Int. 2008;83(6):388-92.

32. Alp A. Effects of aerobic exercise on bone-specific alkaline phosphatase and urinary CTX levels in premenopausal women. Turkish J Phys Med Rehabil. 2013;59(4):310-3.

33. Lutz LJ, Karl JP, Rood JC, Cable SJ, Williams KW, Young AJ, et al. Vitamin D status, dietary intake, and bone turnover in female soldiers during military training. J Int Soc Sports Nutr. 2012;9:1-7.

34. Roghani T, Torkaman G, Movasseghe S, Hedayati M, Goosheh B, Bayat N Effects of short-term aerobic exercise with and without external loading on bone metabolism and balance in postmenopausal women with osteoporosis. Rheumatol Int. 2013;33(2):291-8. 
35. Tajima O, Ashizawa N, Ishii T, Amagai H, Mashimo T, Liu L, et al. Interaction of the effects between vitamin $\mathrm{D}$ receptor polymorphism and exercise training on bone metabolism. J Appl Physiol. 2000;88(8750-7587):1271-6.

36. Vincent KR, Braith RW. Resistance exercise and bone turnover in elderly men and women. Med Sci Sports Exerc. 2002;34(1):17-23.

37. Ardawi M-SM, Rouzi AA, Qari MH. Physical activity in relation to serum sclerostin, insulin-like growth factor-1, and bone turnover markers in healthy premenopausal women: a cross-sectional and a longitudinal study. J Clin Endocrinol Metab. 2012;97(10):3691-9.

38. Erickson CR, Vukovich MD. Osteogenic index and changes in bone markers during a jump training program: a pilot study. Med Sci Sports Exerc. 2010; 42(8):1485-92.

39. Fujimura R, Ashizawa N, Watanabe M, Mukai N, Amagai H, Fukubayashi T, et al. Effect of resistance exercise training on bone formation and resorption in young male subjects assessed by biomarkers of bone metabolism. J Bone Miner Res. 1997;12(4):656-62.

40. Basat $\mathrm{H}$, Esmaeilzadeh S, Eskiyurt N. The effects of strengthening and highimpact exercises on bone metabolism and quality of life in postmenopausal women: a randomized controlled trial. J Back Musculoskelet Rehabil. 2013; 26(4):427-35.

41. Menkes A, Mazel S, Redmond R, Koffler K, Libanati C, Gundberg C, et al. Strength training increases regional bone mineral density and bone remodeling in middle-aged and older men. J Appl Physiol. 1993;74(5):247884.

42. Schroeder ET, Hawkins SA, Jaque SV. Musculoskeletal adaptations to 16 weeks of eccentric progressive resistance training in young women. J Strength Cond Res. 2004;18(2):227-35.

43. Kelley G, Kelley K. Systematic reviews and meta-analysis in nutrition research. Br J Nutr. 2019;122:1279-94.

44. Moher D, Shamseer L, Clarke M, CHersi D, Liberati A, Petticrew M, et al. Preferred Reporting Items for Systematic Review and Meta-Analysis Protocols (PRISMA-P) 2015 statement. Syst Rev. 2015;4(1). https://doi.org/10. 1186/2046-4053-4-1

45. Clowes JA, Hannon RA, Yap TS, Hoyle NR, Blumsohn A, Eastell R. Effect of feeding on bone turnover markers and its impact on biological variability of measurements. Bone. 2002;30(6):886-90.

46. Babraj JA, Smith K, Cuthbertson DJR, Rickhuss P, Dorling JS, Rennie MJ. Human bone collagen synthesis is a rapid, nutritionally modulated process. J Bone Miner Res. 2005;20(6):930-7.

47. Bjarnason N, Henriksen EE, Alexandersen P, Christgau S, Henriksen D, Christiansen C. Mechanism of circadian variation in bone resorption. Bone. 2002;30(1):307-13.

48. Sale C, Varley I, Jones T, James R, Tang J, Fraser W, et al. Effect of carbohydrate feeding on the bone metabolic response to running. J Appl Physiol. 2015;119(7):824-30

49. Qvist P, Christgau S, Pedersen B, Schlemmer A, Christiansen C. Circadian variation in the serum concentration of C-terminal telopeptide of type I collagen (serum CTx): effects of gender, age, menopausal status, posture, daylight, serum cortisol, and fasting. Bone. 2002;31(1):57-61.

50. Vasikaran S, Eastell R, Bruyère O, Foldes AJ, Garnero P, Griesmacher A, et al. Markers of bone turnover for the prediction of fracture risk and monitoring of osteoporosis treatment: a need for international reference standards. Osteoporos Int. 2011;22(2):391-420.

51. Kouvelioti R, LeBlanc P, Falk B, Ward W, Josse A, Klentrou P. Effects of highintensity interval running versus cycling on sclerostin, and markers of bone turnover and oxidative stress in young men. Calcif Tissue Int. 2019;1(1):1-9.

52. Santos $L$, Elliott-Sale KJ, Sale C. Exercise and bone health across the lifespan. Biogerontology. 2017;18(6):931-46.

53. Fu R, Gartlehner G, Grant M, Shamliyan T, Sedrakyan A, Wilt T, et al. Conducting quantitative synthesis when comparing medical interventions: AHRQ and the Effective Health Care Program. J Clin Epidemiol. 2011;64(11): 1187-97.

54. Lefebvre C, Glanville J, Briscoe S, Littlewood A, Marshall C, Metzendorf M, et al. Searching and selecting studies. In: Higging J, Thomas J, Chandler J, Cumpston M, Li T, Page M, et al., editors. Cochrane handbook for systematic reviews of interventions. Version 6. Cochrane; 2020.

55. Banfi G, Lombardi G, Colombini A, Lippi G. Bone metabolism markers in sports medicine. Sport Med. 2010;40(8):697-714.

56. Alp A. Bone-specific alkaline phosphatase and exercise. In: Preedy V, editor. Biomarkers in bone disease biomarkers in disease: methods, discoveries and applications. Dordrecht: Springer; 2015.
57. van Driel M, De Sutter A, De Maeseneer J, Christiaens T. Searching for unpublished work in Cochrane reviews may not be worth the effort. J Clin Endocrinol Metab. 2009;62(8):838-44.

58. McGowan J, Sampson M, Salzwedel D, Cogo E, Foerster V, Lefebvre C. PRESS Peer Review of Electronic Search Strategies: 2015 guideline statement. J Clin Epidemiol. 2016;75:40-6.

59. Cohen J. Weighted kappa: nominal scale agreement with provision for scaled disagreement or partial credit. Psychol Bull. 1968;70:213-20.

60. Downs S, Black N. The feasibility of creating a checklist for the assessment of the methodological quality both of randomised and non-randomised studies of health care interventions. J Epidemiol Commun ealth. 1998;52(6): 377-84

61. Kruschke J, Liddelll T. The Bayesian new statistics: hypothesis testing, estimation, meta-analysis, and power analysis from a Bayesian perspective. Psychon Bull Rev. 2018;25(1):178-206.

62. Morris S. Estimating effect sizes from pretest-posttest- control group designs. Organ Res Methods. 2008;11(2):364-86.

63. Cohen J. A power primer. Psychol Bull. 1992;112:155-9.

64. Bürkner P. brms: an R package for Bayesian multilevel models using Stan. J Stat Softw. 2017:80(1):1-28.

65. Fernandez-Castilla B, Declercq L, Jamshidi L, Beretvas S, Onghena P, Van den Noorthgate W. Detecting selection bias in meta-analyses with multiple outcomes: a simulation study. J Exp Educ. 2019. https://doi.org/10.1080/ 00220973.2019 .1582470

66. Guyatt G, Oxman A, Vist G, Kunz R, Falck-Ytter Y, Alonso-Coello P, et al. GRADE: an emerging consensus on rating quality of evidence and strength of recommendations. Br Med J. 2008:336(7650):924-6.

\section{Publisher's Note}

Springer Nature remains neutral with regard to jurisdictional claims in published maps and institutional affiliations.

Ready to submit your research? Choose BMC and benefit from:

- fast, convenient online submission

- thorough peer review by experienced researchers in your field

- rapid publication on acceptance

- support for research data, including large and complex data types

- gold Open Access which fosters wider collaboration and increased citations

- maximum visibility for your research: over $100 \mathrm{M}$ website views per year

At $\mathrm{BMC}$, research is always in progress.

Learn more biomedcentral.com/submissions 